\title{
EFFECTS OF PUERARIN ON THE REDUCTION OF GLUCOSE AND PROMOTION OF OVERALL HEALTH IN ACUTELY STRESSED CHINOOK SALMON, ONCORHYNCHUS TSHAWYTSCHA (ACTINOPTERYGII: SALMONIFORMES: SALMONIDAE)
}

\author{
Mohammed Rumman HOSSAIN, Elliott BLUMENTHAL, and Ahmed MUSTAFA*
}

\author{
Indiana University-Purdue University Fort Wayne, 2101 E. Coliseum Blvd, Fort Wayne, IN, USA
}

Hossain M.R., Blumenthal E., Mustafa A. 2013 Effects of puerarin on the reduction of glucose and promotion of overall health in acutely stressed Chinook salmon, Oncorhynchus tshawytscha (Actinopterygii: Salmoniformes: Salmonidae). Acta Ichthyol. Piscat. 43 (2): 85-93.

\begin{abstract}
Background. Farmed fish reared in aquaculture systems are exposed to physiological stressors from biological, chemical, and physical sources. The consequences of these stressors affect the productivity of farming outcomes by reducing immune response, growth, and development within fish. A 24-h study was performed to determine the effects of supplementing puerarin - the most biologically active ingredient present in the roots of kudzu (Pueraria lobata) — within farmed fish on acute handling stress.

Materials and methods. Fingerlings of Chinook salmon, Oncorhynchus tshawytscha, were maintained at $16-18^{\circ} \mathrm{C}$ in 10 US gallon $(37.8 \mathrm{~L})$ glass tanks, supplied with recirculated and aerated dechlorinated water. Puerarin was given to fish through supplementation to commercial fish feed pellets. Acute handling stress was induced through transferring the fish from their housing tanks into 8 designated tanks at $0000 \mathrm{~h}$. Subsequently, at $5 \mathrm{~h}$ post-transfer all the fish were fed to satiation, following which the stressed groups were chased with nets. Results. The results indicated that administration of puerarin through feed supplementation reduces plasma glucose, hematocrit, plasma protein, and improves spleen somatic indices after $24 \mathrm{~h}$, and thereby counteracting the negative consequences of acute stress induced through handling.

Conclusion. The results suggest that supplementation of puerarin to commercial fish feed improved overall fish health through enhancing systemic circulation while attenuating plasma glucose. Being one of the first studies to study the effects of puerarin on fish, our results are in agreement with prior research with puerarin on other animal models.
\end{abstract}

Keywords: salmon, aquaculture, stress, puerarin

\section{INTRODUCTION}

In the realm of aquaculture, the loss of productivity through infection and subsequent reductions in growth and development is a major problem which requires innovative methods for generating solutions. There are no commercially available vaccines against many aquatic pathogens, and further exacerbating the problem, the use of antibiotics for treatment of the fish is not preferred due to the risk of developing antibiotic resistance within the bacteria, harming non-target species, and the potential of developing zoonotic parasites which can infect human populations from farmed sources (Hadidi et al. 2008). Further complicating the situation is the fact that in aquaculture, fish are raised in tanks with thousands of other fish under crowded conditions, which can negatively affect feeding behavior, along with impacting other normal physiological functions such as growth, immune response, reproductive prowess (Barton and Iwama 1991). According to the life-history theory, each organism must allocate its energy within several necessary activities: survival, reproduction, and somatic maintenance (Poisot et al. 2009). Since each organism has a limited amount of energy remaining after performing all the necessary activities at its disposal, a pathogenic infection can easily disrupt the homeostatic functions within poikilotherms such as fish. This is because mounting an effective immune response is a highly energetically expensive process (Poisot et al. 2009). Thus, the manifestation of stress in farmed fish through common industry practices such as crowding or handling for vaccination, grading, transportation, etc. in aquaculture makes farmed fish more susceptible to parasitic infections as they possess reduced capacities to mount effective immune responses (Barton and Iwama 1991, Ruane et al. 1999).

Within the aquaculture industry, the protocols for handling fish are well-documented acute stressors, which

\footnotetext{
${ }^{*}$ Correspondence: Dr. Ahmed Mustafa, Department of Biology, India University-Purdue University Fort Wayne, Fort Wayne, IN 46805, USA, phone: +1-260-48-6328; e-mail: mustafaa@ipfw.edu.
} 
lead to stress levels which are tolerated despite causing undesirable effects (Gerwick et al. 1999). In fish, the stress response is marked by the stimulation of the hypothalamuspituitary-interrenal (HPI) axis, which leads to an increment in the levels of plasma cortisol and the sympathetic-catecholamine cell axis which elevates catecholamine levels (Wendelaar Bonga 1997, Mustafa et al. 2000). Cortisol is an immunosuppressive agent, and increased occurrences of diseases have been observed within stressed fish (Barton and Iwama 1991, Leonard and McCormick 1999, Ruane et al. 1999). In addition to a suppressed immune response through cortisol, acute stress has been characterized by hyperglycemia, which has been associated with complications such as strokes in both animal and human models (Capes et al. 2001), along with increased susceptibility to infections in fish (Barton and Iwama 1991). Teleost fish have been well documented for their reduced capacities to clear plasma glucose levels, and hence are more prone to suffer from stress-induced hyperglycemia (Moon 2001).

In mammalian tissues, the glucose transporter (GLUT)-4 is the primary glucose transporter that is expressed in insulin-sensitive issues such as adipose tissue, skeletal muscle, and heart (Díaz et al. 2007). This transporter performs an integral task in the homeostasis of glucose by allowing insulin to function via the increase in glucose uptake within the insulin-responsive tissues (Díaz et al. 2007). Under normal cellular conditions, GLUT-4 is present within intracellular stores until it is stimulated by insulin, which causes the transporter protein to be translocated from the cytoplasm to the plasma membrane, thus allowing glucose to enter into the cells stimulated by insulin (Díaz et al. 2007). Hence, GLUT-4 has been considered a crucial component in avoiding plasma hyperglycemia, such as during the postprandial period, or during times of stress (Díaz et al. 2007). Furthermore, Díaz et al. (2007) reveal that while rainbow trout muscle tissue does possess a GLUT-4 transporter which is similar in structure and function to the GLUT-4 transporter proteins found in the skeletal muscle tissues present in mice, the marine homolog of GLUT-4 is less efficient than the murine version in clearing plasma glucose.

Therefore, this study was developed to determine the effects of puerarin, the most biologically active isoflavone present in the roots of kudzu (Pueraria lobata) (see Prasain et al. 2004), which is an invasive plant (Peng et al. 2009), on modulating acute stress within salmonids. Since salmonids are known for their migration patterns upstream, a practice that has been reported to induce stress within fish (Leonard and McCormick 1999). While kudzu powder has been used in oriental countries as a natural treatment for cardiovascular diseases (Yan et al. 2006), puerarin has been shown to reduce skeletal tissue blood glucose levels in diabetic mice over a period of 1 day; moreover, puerarin helped restore GLUT-4 mRNA expression levels in the diabetic mice to those of non-diabetic mice within a duration of 3 days (Hsu et al. 2003). Since rainbow trout, Oncorhynchus mykiss (Walbaum, 1792), possessed GLUT-4 transporter proteins, we chose chi- nook salmon (also called king salmon), Oncorhynchus tshawytscha (Walbaum, 1792), which belongs to the same genus as the rainbow trout, and is a species which is farmed. In addition, chinook salmon has been shown to elicit very high post-stress plasma cortisol levels; often reaching $400 \mathrm{ng} \cdot \mathrm{mL}^{-1}$ during upstream migration in the wild (Barton 2002). Performing stress response studies under laboratory conditions are difficult since most often the fish are acquired from hatcheries where fish are reared with thousands of other fish. Therefore, it is harder to induce stress through crowding as the fish are accustomed to be in close proximity to each other since birth. Thus, fingerling chinook salmon, due to their capacity to be stressed, provided us with a good model organism to determine the effects of puerarin on acute stress, which can be difficult to elicit amongst other fish species (Barton 2002). In addition, we hypothesized that since crowding is ubiquitous in aquaculture, the major disruptive practice of handling or chasing for transportation or vaccination is more unsettling as they weaken the animals to a greater extent. Therefore, we decided to induce stress through handling, since we could also be certain of the generation of stress within the fish.

We hypothesized that since teleost fish possess diminished capacities to clear their blood-glucose levels, the presence of puerarin will help alleviate the symptoms of elevated sugar concentrations within the organisms. Subsequently, this will allow the fish to regain normal homeostatic levels which will signal the cessation of other stimulators of the stress response such as cortisol. The aim of this study was to determine the impact of puerarin on physiological parameters such as plasma cortisol, blood-glucose, plasma protein levels, packed-cell volume, and spleen somatic indices (SSIs), which has been studied in multiple fish-stress studies (Barton and Iwama 1991, Wendelaar Bonga 1997, Ruane et al. 1999, Hadidi et al. 2008).

\section{MATERIALS AND METHODS}

Fish acquisition and maintenance. Chinook salmon fingerlings ( 3 months old) weighing between 2 to $5 \mathrm{~g}$ were obtained from the Bodine State Fish Hatchery (Mishawaka, IN) and transported to the Life Sciences Resource Center (LSRC) at Indiana University-Purdue University Fort Wayne. The fish were housed in twentyfour 10 US gallon $(37.8 \mathrm{~L})$ tanks containing dechlorinated water which was filtered and oxygenated by Millennium 2000 filters (Aquarium Systems, OH). The dechlorination stage consisted of filling large bins with city water; bins were left uncovered under light for approximately one week with aeration. Through this process chlorine was slowly evaporated from the supplied water in the bins, which was subsequently used to fill the fish tanks. After being transported into LSRC, the fish were allowed to acclimate to their new conditions for two months. The fish were fed daily with commercial fish feed (Aquamax Starter Fish Fry 200) — which would form the control feed for our study - to satiation, while being observed routinely to prevent any manifestation of any disease. 
The water temperature was monitored once daily during the acclimation period and twice daily within the acute stress study. The fish were housed in a room where the ambient temperature did not exceed $18^{\circ} \mathrm{C}$ and the water temperature for the fish was kept between $16-18^{\circ} \mathrm{C}$. In addition to water temperature, water chemistry parameters such as dissolved oxygen of the water were kept in between 6.5 and $9.3 \mathrm{mg} \cdot \mathrm{L}^{-1}$, and the $\mathrm{pH}$ in between 6 and 9 . In addition, the ammonia was less than $0.25 \mathrm{mg} \cdot \mathrm{L}^{-1}$, the nitrate in within $25-50 \mathrm{mg} \cdot \mathrm{L}^{-1}$, and the nitrite at $0.3 \mathrm{mg} \cdot \mathrm{L}^{-1}$. All of the aforementioned parameters were within the prescribed values from Wedemeyer et al. (1990). Throughout the acclimation and study period, the fish were kept in a room with a diurnal cycle which was divided into $16 \mathrm{~h}$ light and $8 \mathrm{~h}$ of darkness.

During the study, the control fish were fed control feed, while the puerarin-feed fish were fed Aquamax Starter Fry 200 fish feed which was supplemented with puerarin prior to the start of the study. Puerarin supplement (catalogue \# P5555) was purchased from SigmaAldrich (St. Louis, MO). To incorporate puerarin into the control feed, the isoflavone was dissolved in a solution of reagent grade $95 \%$ alcohol due to puerarin's high solubility in alcohol (Wang and Cheng 2005). The dissolved solution of puerarin was then carefully sprayed on the feed in small aliquots, and the feed was air-dried and stored at $4^{\circ} \mathrm{C}$ (Mustafa and MacKinnon 1999) to make a concentration of $200 \mathrm{mg} \cdot \mathrm{kg}^{-1}(0.02 \%)$. The concentration was deemed appropriate since prior studies on mice by Peng et al. (2009) used feed which gave mice $200 \mathrm{mg}$ kudzu powder which consisted of $68 \mathrm{mg}$ of puerarin. Our feed consisted of $200 \mathrm{mg}$ of puerarin, which is approximately three times greater than Peng et al. (2009), since we wanted to ensure that the fish were getting enough puerarin, as there is wastage when puerarin is thrown in the water. To minimize the loss of puerarin through evaporation, the feed was prepared within one week prior to the start of the study in June.

Experimental design. On the day of the start of the study at $0000 \mathrm{~h}$, stress was induced by randomly transferring all the fish to eight tanks located in two separate systems: the first system contained four tanks: two of which housed the control unstressed groups while the remaining two tanks housed the control stressed group. Similarly, the second system also consisted of four tanks which were fed puerarin-supplemented feed: two tanks housed fish which were not stressed, while the remaining two consisted of fish which were stressed. The separation of the systems was done to prevent any cross-contamination of feed between the control and puerarin feed groups. This is because the systems used for this study recycled the majority of the water that ran through the tanks. Prior to the random distribution of fish into the experimental groups, 6 fish were randomly selected from the 24 tanks and were sampled for physiological and immunological parameters and accounted for our results from day 0 (CFU D0). Five $\mathrm{h}$ following the transfer, the fish were fed feeds according to their respective groups. Shortly after the fish were fed to satiation, stress was induced within the stressed groups by chasing, catching, and netting the fish with a net for a period of $5 \mathrm{~min}$ to induce acute handling stress. Subsequently, the fish were sampled $24 \mathrm{~h}$ after being transferred to their respective tanks.

Two samplings were performed for this study: at $0000 \mathrm{~h}$ and $2400 \mathrm{~h}$. All the sampled fish were selected randomly and caught efficiently with the least amount of chasing with a net. Following capture, the fish were euthanized in a $1 \mathrm{~L}$ solution of $200 \mathrm{mg}$ of MS-222 (tricane methanosulfonate; Sigma-Aldrich; St. Louis, MO) (Gerwick et al. 1999, Halloway et al. 2004). Subsequently, the health and stress levels of the fish were determined by the measuring the length, weight, condition factor $(\mathrm{K})$, blood glucose, hematocrit, plasma protein levels, and spleen sizes to calculate spleen somatic indices (SSIs).

The 4 experimental groups in this experiment were categorized as control feed unstressed (CFU), control feed stressed (CFS), puerarin/kudzu feed unstressed (KFU), and puerarin/kudzu feed stressed (KFS). Since the study was performed over a 24-h period, the control feed unstressed fish at the start of the experiment or at $0000 \mathrm{~h}$ were categorized as CFU D0, while the remaining groups were sampled after $24 \mathrm{~h}$ or 1 day, which are classified as D1. Therefore, the 4 sampled groups at day 1 were labeled as CFU D1, CFS D1, KFU D1, and KFS D1.

Blood collection. The fish were sampled by drawing blood from the caudal vein with the help of heparinized syringes to prevent clotting. The needles were inserted into the midline of the fish while aiming for the center of the vertebral body. The extracted blood samples were then utilized for determining the plasma cortisol, plasma glucose, hematocrit, and total protein levels.

Plasma cortisol and glucose concentration measurements. Cortisol concentration was determined with the help of the goat antibody kit using plasma cortisol collected from fish sampled. The kit was purchased from Enzo Life Sciences, Farmingdale, NY. The measurements are expressed in $\mathrm{ng} \cdot \mathrm{mL}^{-1}$ with a sensitivity of less than 1 percentage point. The minimum detectable level of cortisol for the kit was $0.156 \mathrm{ng} \cdot \mathrm{mL}^{-1}$.

Plasma glucose concentration was determined by placing a drop of collected blood from each fish onto a glucometer strip which was then placed in a standard glucometer (Precision Xtra, Abbott Laboratories; Abbott Park, IL). This method for testing blood glucose has been validated for use on fish by Wedemeyer et al. (1990).

Blood hematocrit and plasma protein levels. Following plasma glucose measurement, blood hematocrit levels were measured from the collected blood when loaded into a capillary tube. After loading, the capillary tubes was capped with at one end and spun in micro-centrifuge. After spinning, Micro-Hematocrit Capillary Tube Readers (Monoject Scientific, St. Louis, MO) were employed to determine Packed Cell Volume (PCV). Subsequently, plasma protein levels were measured through the usage of refractometer after adding one to two drops from the spun hematocrit tubes. 
Spleen somatic indices (SSI). SSI were generated when the spleen was removed from each sampled fish and weighed on an electrical scale. Upon weighing, the SSI was calculated through the usage of the formula: SSI $=($ spleen weight/ body weight $) \times 100($ Pearson and Stevens 1991) .

Statistical analyses. The data collected through the course of this experiment were analyzed using SigmaPlot 11.0 software. The means and standard errors of the means were determined for each assay through one-way analysis of variance (ANOVA). Subsequently Tukey's test and Student's simple $t$-test were performed to determine whether the differences between the samples were significant $(P<0.05)$. The findings of our analyses are presented in the text and graphs in the form of means \pm standard errors of means (SEM).

Ethical issues. Fish were kept at optimal aquatic conditions set for this species throughout the duration of the experiment and cared for according to the guidelines of the Purdue University Animal Care and Use Committee.

\section{RESULTS}

Over 24-h period, the plasma cortisol concentrations of the unstressed groups within both feed groups receded to levels similar to those of from day 0 . On $0000 \mathrm{~h}$ (D0), the cortisol concentration for control feed unstressed (CFU) was $\left(2.29 \pm 0.31 \mathrm{ng} \cdot \mathrm{mL}^{-1}\right)$, which was similar to that of puerarin feed unstressed (KFU) $\left(2.01 \pm 0.41 \mathrm{ng} \cdot \mathrm{mL}^{-1}\right)$ after $24 \mathrm{~h}$ (D1), while control feed unstressed at day 1 (CFU D1) underwent a cortisol reduction at $(1.14 \pm 0.41$ $\left.\mathrm{ng} \cdot \mathrm{mL}^{-1}\right)$ which was significantly different $(P<0.05)$ from both CFU D0 and KFU D1 (Fig. 1). Whereas both unstressed groups had their plasma cortisol levels revert back to basal levels after $24 \mathrm{~h}$, both stressed groups (control feed stressed (CFS) and kudzu feed stressed (KFS)) experienced increases in circulating concentrations of cortisol. The stressed groups fed control diet (CFS D1) elicited cortisol levels $\left(10.83 \pm 5.34 \mathrm{ng} \cdot \mathrm{mL}^{-1}\right)$ which were approximately ten times greater than unstressed control feed. Conversely, puerarin fed stressed group (KFS D1) produced cortisol levels $\left(14.67 \pm 3.42 \mathrm{ng} \cdot \mathrm{mL}^{-1}\right)$ which was approximately seven times greater than that of KFU D1 and significantly different $(P<0.05)$ from CFU D1. Therefore, the experimental conditions were successful in manifesting stress, which can be inferred from the elevated levels of cortisol within the stressed groups relative to the unstressed groups.

The blood glucose concentrations within the four groups varied depending on the type of feed, rather than the type of stress treatment being administered. Whereas differences in plasma cortisol concentrations could be distinguished based on the cumulative nature of the stressors applied, blood glucose levels were similar within the stressed and non-stressed groups (Fig. 2). However, the differences between the basal values from $0000 \mathrm{~h}$ (CFU D0) $\left(45.33 \pm 8.09 \mathrm{mg} \cdot \mathrm{dL}^{-1}\right)$ were significantly different $(P<0.05)$ from those of CFU D1 $\left(79 \pm 9.7 \mathrm{mg} \cdot \mathrm{dL}^{-1}\right)$ and CFS D1 $\left(70 \pm 3.93 \mathrm{mg} \cdot \mathrm{dL}^{-1}\right)$ after $24 \mathrm{~h}$. Therefore, the plasma glucose concentrations within both stressed (CFS D1) and non-stressed (CFU D1) control feed groups were significantly elevated relative to the fish from day 0 (CFU D0). Similarly, the plasma glucose concentrations for KFU D1 $\left(42.67 \pm 4.26 \mathrm{mg} \cdot \mathrm{dL}^{-1}\right)$ was significantly lower $(P<0.05)$ than both CFU D1 and CFS D1. However, there were no significant differences between CFU D1 and CFS D1. Conversely, KFS D1 $\left(49.67 \pm 5.97 \mathrm{mg} \cdot \mathrm{dL}^{-1}\right)$ was significantly lower $(P<0.05)$ than CFS D1. Meanwhile, the difference between CFU D1 and KFS D1was approximately 37 percentage points, and there were no significant differences between KFU D1 and KFS D1. Therefore, there were significant differences between control-feed unstressed and puerarin-feed stressed and unstressed groups.

Hematocrit or packed cell volumes (PCVs) were calculated to be elevated across all four groups after $24 \mathrm{~h}$ (Fig. 3). On day 0 , the basal value (CFU D0) was $(33.75 \% \pm 1.25 \%)$, which was significantly lower $(P<0.05)$ than both CFU D1 $(43.33 \% \pm 1.20 \%)$ and CFS D1 $(47.33 \% \pm 4.49 \%)$ after $24 \mathrm{~h}$. There was no significant difference between CFU D1 and CFS D1. Therefore, both control feed groups produced higher PCVs than that of CFU D0. In addition, CFU D0 was also significantly lower $(P<0.05)$ than KFS D1 $(40.00 \% \pm 1.73 \%)$. While there is no significant difference between KFU D1 $(31.67 \% \pm 4.91 \%)$ and KFS D1, the former was 20 percentage points lower than CFU and 33 percentage points lower than CFS.

Plasma protein levels were elevated within all four groups on day 1 relative to the levels from day 0 (CFU D0) $\left(3.33 \pm 0.17 \mathrm{~g} \cdot \mathrm{dL}^{-1}\right)$, which was significantly lower $(P<0.05)$ than both CFU D1 $\left(4.93 \pm 0.18 \mathrm{~g} \cdot \mathrm{dL}^{-1}\right)$ and CFS D1 (5.27 $\left.\pm 0.33 \mathrm{~g} \cdot \mathrm{dL}^{-1}\right)$ (Fig. 4). Therefore, both control feed groups: stressed and unstressed, had greater plasma protein in their blood. Similarly, CFU D0 had significantly lower $(P<0.05)$ plasma protein concentration than KFU D1 $\left(4.07 \pm 0.07 \mathrm{~g} \cdot \mathrm{dL}^{-1}\right)$, which was significantly lower $(P<0.05)$ than both CFU and CFS at day 1 . Therefore, the supplementation of puerarin lowered plasma protein concentrations within the unstressed group. There was no significant difference between KFS D1 $\left(4.27 \pm 0.37 \mathrm{~g} \cdot \mathrm{dL}^{-1}\right)$ and KFU D1, and KFS D1 about 6 percentage points higher than KFU D1, while being approximately 15 percentage points lower than CFU D1 and approximately 23 percentage points lower than CFS D1. Therefore, fish fed puerarin had improved markers which can be used to predict improved blood flow within fish.

Our findings indicate that the transfer of salmon into specific groups was a stressor, since spleen sizes were reduced in all four groups relative to the values obtained from day 0 . However, the spleen sizes were further reduced within both stressed groups relative to both control groups (CFU D1 and CFS D1) after 24 h; conversely, spleen sizes were slightly bigger within puerarin feed stressed groups (Fig. 5). The SSIs for CFU D0 $(0.24 \pm 0.019)$ was significantly higher $(P<0.05)$ than CFS D1 $(0.12 \pm 0.018)$ and KFS D1 $(0.15 \pm 0.016)$. The difference between KFU D1 $(0.17 \pm 0.008)$ and KFS D1 was 12 percentage points while the difference between CFU D1 $(0.18 \pm 0.016)$ and CFS D1 was 31 percentage points. Overall, SSIs were 
approximately 20 percentage points lower within control feed unstressed (CFU) and puerarin feed unstressed (KFU) from D1 relative to control feed unstressed at day 0 (CFU D0). Therefore, the cumulative effects of moving into different tanks and the subsequent chasing with nets produced similar shrinkage of spleens within the fish. However, the puerarin feed stressed group appears to have coped marginally better with the stressful stimuli than control feed stressed group.

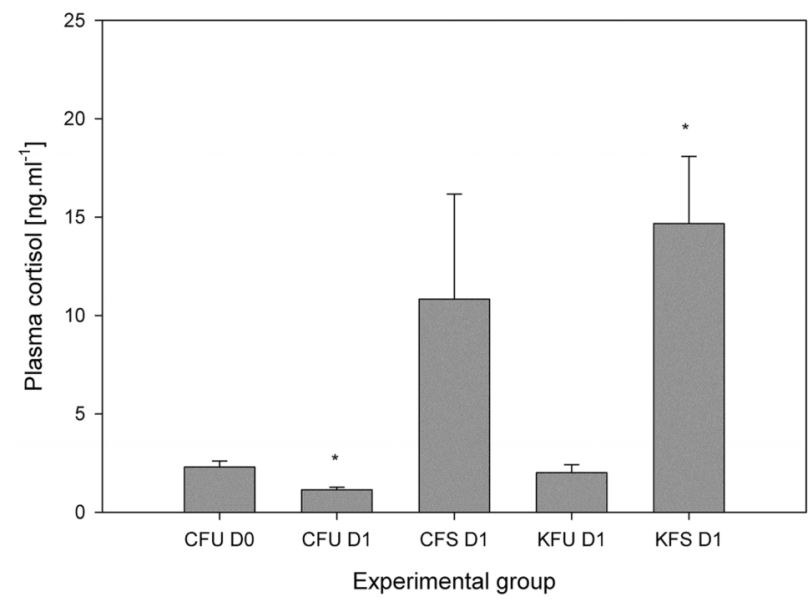

Fig. 1. Plasma cortisol concentrations (mean \pm SEM) in different groups of experimental fish (Chinook salmon, Oncorhynchus tshawytscha); CFU = control feed, not stressed; CFS = control feed, stressed; KFU $=$ puerarin feed, not stressed; KFS $=$ puerarin feed, stressed; D0 $=0 \mathrm{~h}$ per day $0 ; \mathrm{D} 1=24 \mathrm{~h}$ per day 1 ; *significantly different $(P<0.05)$ from CFU D0 (Control feed not stressed group at day 0$)$

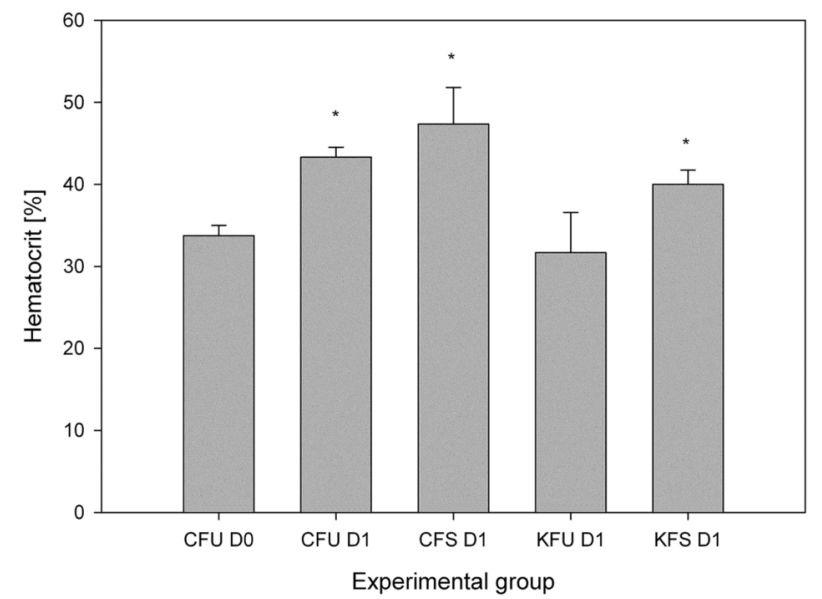

Fig. 3. Hematocrit or Packed Cell Volume (PCV) (mean \pm SEM) in different groups of experimental fish (Chinook salmon, Oncorhynchus tshawytscha); CFU $=$ control feed, not stressed; CFS $=$ control feed, stressed; KFU = puerarin feed, not stressed; KFS = puerarin feed, stressed; D0 $=0 \mathrm{~h}$ per day $0 ; \mathrm{D} 1=24 \mathrm{~h}$ per day 1 ; *significantly different $(P<0.05)$ from CFU D0 (Control feed not stressed group at day 0 )

\section{DISCUSSION}

The production of cortisol and its subsequent release from interrenal cells (adrenal cortex homologue in fish) within the HPI axis takes several minutes, and research has shown that proper execution of protocol is sufficient in allowing the measurement of resting levels of cortisol within fish (Barton 2002). However, the practice of preparing fish for experimental handling by capturing and transferring them to a small container consisting of anesthetizing or

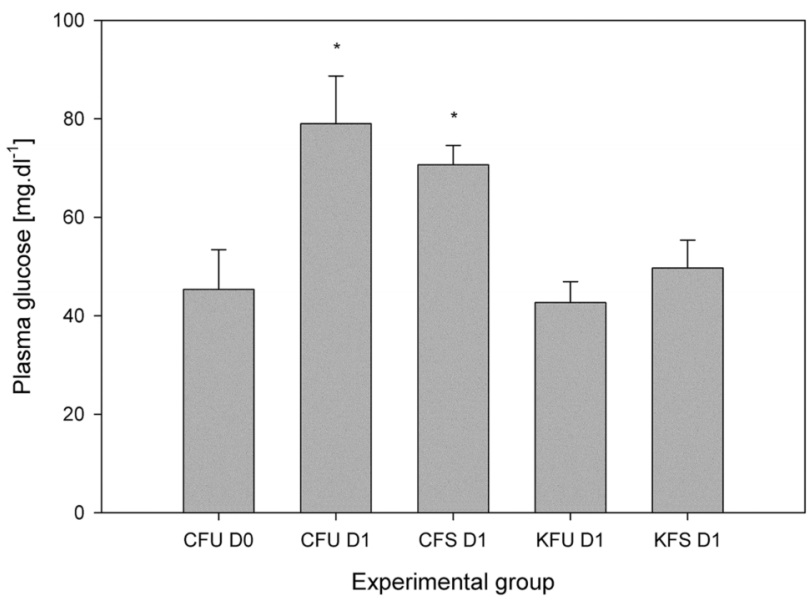

Fig. 2. Plasma glucose concentrations (mean \pm SEM) in different groups of experimental fish (Chinook salmon, Oncorhynchus tshawytscha); CFU = control feed, not stressed; CFS = control feed, stressed; KFU $=$ puerarin feed, not stressed; KFS = puerarin feed, stressed; D0 $=0$ h per day $0 ; \mathrm{D} 1=24 \mathrm{~h}$ per day 1 ; *significantly different $(P<0.05)$ from CFU D0 (Control feed not stressed group at day 0 )

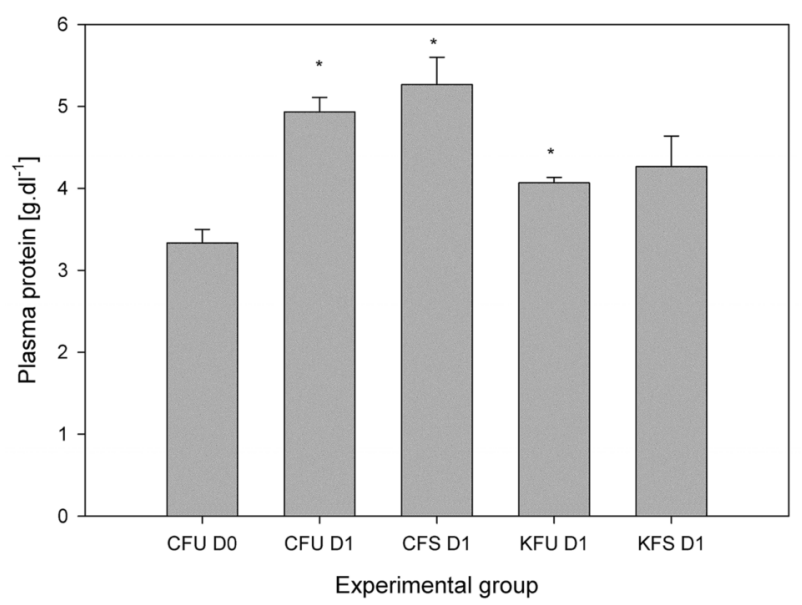

Fig. 4. Plasma protein concentrations (mean \pm SEM) in different groups of experimental fish (Chinook salmon, Oncorhynchus tshawytscha); CFU = control feed, not stressed; CFS = control feed, stressed; KFU $=$ puerarin feed, not stressed; KFS $=$ puerarin feed, stressed; D0 $=0 \mathrm{~h}$ per day 0 ; D1= $24 \mathrm{~h}$ per day 1 ; *significantly different $(P<0.05)$ from CFU D0 (Control feed not stressed group at day 0 ) 
euthanizing agent such as MS-222 (tricaine methanesulfonate) has deficiencies; since this practice exposes the fish to the stress of being chased, netted, passed through the air, placed into a small, unfamiliar container before the anesthetic takes effect (Gerwick et al. 1999). The latter procedure, even when executed proficiently, cannot eliminate the possibility of the activation of sympathetic stress response pathways (Gerwick et al. 1999). Thereby, increasing the chances of variation within the sampled fish and reducing the possibility of acquiring the most accurate data. On the other hand, one can counteract such an obstacle by sampling more fish; however, the chances of induction of the stress response within both the stressed and unstressed groups increases each time attempts are made to capture more fish. Therefore, near the end of a long sampling run, most of the fish will be stressed and have elicited sympathetic activation; thus, producing inaccurate results (Holloway et al. 2004). Therefore, the sample sizes were kept relatively low in order to reduce the chances of sympathetic activation. Such restrictions highlight the necessity of the development of new techniques in the practice of capturing fish for sampling.

After being transferred to new tanks, $24 \mathrm{~h}$ was sufficient for returning plasma cortisol levels to the basal level from day 0 , since both CFU D1 and KFU D1 produced similar concentrations of cortisol, which is in agreement from prior research studies (Barton and Peter 1982, Barton 2002). Also, the practice of chasing the stressed groups with nets for 5 min was successful in eliciting activation of the HPI axis as both CFS D1 and KFS D1 produced similarly elevated plasma cortisol levels after $24 \mathrm{~h}$. In addition to the potential of variations resulting from the capture protocol, other factors such as heritability or the environment, such as the time of the day, can lead to variations within sampled fish (Bry 1982). Furthermore, due to the dynamic nature of the stress response, multiple physiological parameters are measured due to the mutually exclusive nature of these indicators. The measurement of elevated plasma cortisol concentrations does not necessarily mean that the plasma glucose levels will also be elevated (Barton 2002). Therefore, while our findings illustrate that plasma cortisol levels were attenuated within both unstressed groups, and elevated within both stressed groups, one cannot, however, use our results as a definite indicator for cortisol modulation through both control and puerarin-supplemented feed.

Whereas plasma cortisol is often used as an indicator of the primary stress response (Barton and Iwama 1991, Wendelaar Bonga 1997), the determination of plasma glucose is a useful tool for determining the secondary stress response due to its ease of implementation and widespread usage within aquaculturists (Wedemeyer et al. 1990, Barton 2000). Despite daily fluctuations in plasma glucose levels within salmonids (Barton 2000, 2002), explaining the variations within the two control groups on day 1; surges in plasma glucose concentrations within stressed fish are often used as hallmark indicators of the stress response within salmonids (Wedemeyer et al. 1990,

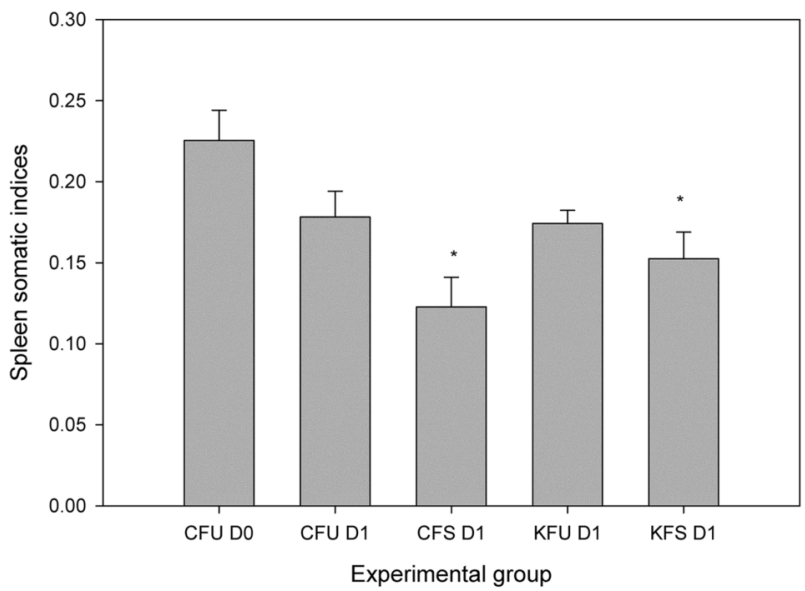

Fig. 5. Spleen somatic indices $(\mathrm{SSI})($ mean $\pm \mathrm{SEM})$ in different groups of experimental fish (Chinook salmon, Oncorhynchus tshawytscha); CFU = control feed, not stressed; CFS = control feed, stressed; $\mathrm{KFU}=$ puerarin feed, not stressed; KFS = puerarin feed, stressed; D0 $=0$ h per day 0 ; D1 $=24$ h per day 1 ; *significantly different $(P<0.05)$ from CFU D0 (Control feed not stressed group at day 0$)$

Barton and Iwama 1991, Wendelaar Bonga 1997). In addition, prior research with puerarin has shown that puerarin improves glucose tolerance and acts as an antihyperglycemic within mice (Meezan et al. 2005). Furthermore, puerarin has been shown to induce increased cellular reuptake of glucose into cells grown in vitro (Hsu et al. 2003). Therefore, our findings, which indicate that the supplementation of puerarin to commercial fish feed attenuates plasma glucose concentrations to levels similar to the glucose concentrations seen in day 0 samples is concurrent with prior research on glucose homeostasis (Hsu et al. 2003). Although our findings could not illustrate any significant differences within both stressed- and unstressed groups fed either control or puerarin feed, our study reveals that puerarin-fed fish had lower plasma glucose levels relative to fish fed control feed. We believe the supplemented puerarin enhances plasma glucose reuptake within the cells of the fish; regardless of stress. This data is concurrent with other studies on the effects of puerarin on insulin-resistant obese mice with reduced glucose tolerance, where puerarin enhanced plasma glucose clearance and reduced plasma insulin levels-reducing the chances of developing insulin resistance within peripheral tissues (Meezan et al. 2005, Prasain et al. 2012). The beneficial effects of enhanced plasma glucose metabolism by puerarin could be observed in other biomarkers of overall health. Since puerarin has also been shown to reduce bone loss and blood cholesterol, all while improving blood circulation and overall health (Meezan et al. 2005, Yan et al. 2006, Peng et al. 2009, Tanaka et al. 2011). Therefore within a 24-h period, we can ascertain that the secretion of cortisol into the blood of our fish led to an elevation of glucose concentration within all four groups. However, while the 
cortisol levels dropped within both control-feed and puerarin-feed unstressed groups, due to the slower glucose homeostasis within fish (Moon 2001, Díaz et al. 2007), the plasma glucose concentrations remained elevated within the control-feed groups, while returning to their physiologically normal levels within the puerarin-feed groups.

While plasma cortisol and glucose are endocrine indicators of stress, hematocrit or packed cell volume (PCV) and plasma protein concentrations are indicative of the cellular response to stress, where $\mathrm{PCV}$ is the ratio of the volume occupied by packed blood cells to the volume of whole blood as measured by a hematocrit (Chaplin et al. 1953). The measurement of hematocrit has been used as an indicator for increased risk and development of cardiac disease (Ring et al. 2008). Upon the induction of acute stress, cells produce proteins such as heat shock proteins and chaperones (Iwama et al. 1999). In addition, the presence of acute mental stress can lead to an increase in PCV and combined with elevated plasma proteins, can cause reductions in blood plasma volume (Ring et al. 2008). A reduction in blood plasma volume will lead to greater energy expenditure by fish to pump blood to their tissues, therefore leading to elevated plasma glucose levels and in extreme cases, exhaustion (Barton and Iwama 1991, Wendelaar Bonga 1997). Furthermore, stress induced hyperglycemia can lead to insulin-resistance which can reduce blood flow within skeletal tissues (Laakso et al. 1990). Our results indicate that the presence of puerarin in fish feed reduced both PCV and plasma protein levels, which is in accord with the findings from studies on other animal models (Yan et al. 2006), in that along with its antihyperglycemic properties, puerarin has been shown to induce vascular relaxation in arteries while enhancing greater blood flow through induction of the synthesis of endothelial nitrous oxide synthase within arteries, and offered protection against hypercholesterolemia within mice (Yeung et al. 2006). In addition, elevated plasma glucose levels have been associated with reduced coronary blood flow (Yokoyoma et al. 1998). Therefore, combining our results with prior findings, puerarin promotes improved blood flow and cardiovascular function, both by relaxing the arterial walls and helping maintain a healthy blood volume, and improves overall health of fish under stress.

In addition to endocrine and cellular markers for stress, the sizes of spleens in fish are used as a predictor of disease resistance (Barton and Iwama 1991, Wendelaar Bonga 1997). In mammals, the spleen is responsible for filtering blood and removing damaged or dying cells which are not fit for circulation (Hadidi et al. 2008). The spleen is specialized for this task since it possesses a dense interlocking web of splenic cords, which contains macrophages which remove any foreign organisms very efficiently when circulating blood runs through (Bohnsack and Brown 1986, Tablin et al. 2002). In case of salmonids, the spleen produces the majority of antibodies and helps form immunological memory (Hadidi et al. 2008). Therefore, the spleen is an important organ, and a smaller spleen is indicative of stress and reduced disease resist- ance, as there is less blood circulating through the spleen, as it is diverted to skeletal tissues for fight or flight response, and there is reduced macrophage activation to counteract any infection (Barton and Iwama 1991, Wendelaar Bonga 1997, Hadidi et al. 2008). In line with prior research, our results indicate that the addition of puerarin assists in enhanced blood flow, which helps in reduced shrinking of the spleen. Therefore, with increased blood flow, macrophages in the spleen will have greater exposure to any pathogens; thereby reducing the need for generating greater numbers of macrophages (an energy extensive process), and henceforth, conserving energy (Barton and Iwama 1991, Wendelaar Bonga 1997, Hadidi et al. 2008).

In conclusion, we were successful in creating a study which generated stressful conditions, under which puerarin, concurring with prior studies, was able to promote overall well being of the fish as it enhanced greater systemic circulation while attenuating plasma glucose. We consider this study informative in illustrating the possibilities with supplementing puerarin with aquaculture feed to improve circulation within fish. However, due to genetic and environmental variations, future studies need to be conducted to determine the cellular interaction with puerarin within fish cells. In addition, subsequent studies plan on evaluating the long-term effects of puerarin-feed on salmonids under stress.

\section{REFERENCES}

Barton B.A. 2000. Salmonid fishes differ in their cortisol and glucose responses to handling and transport stress. North American Journal of Aquaculture 62 (1): 12-18. DOI: 10.1577/1548-8454(2000)062<0012:SFDITC $>2.0 . C O ; 2$

Barton B.A. 2002. Stress in fishes: A diversity of responses with particular reference to changes in circulating corticosteroids. Integrative and Comparative Biology 42 (3): 517-525. DOI: $10.1093 / \mathrm{icb} / 42.3 .517$

Barton B.A., Iwama G.K. 1991. Physiological changes in fish from stress in aquaculture with emphasis on the response and effects of corticosteroids. Annual Review of Fish Diseases 1: 3-26. DOI: 10.1016/0959-8030(91)90019-G

Barton B.A., Peter R.E. 1982. Plasma cortisol stress response in fingerling rainbow trout, Salmo gairdneri Richardson, to various transport conditions, anaesthesia, and cold shock. Journal of Fish Biology 20 (1): 39-51. DOI: 10.1111/j.10958649.1982.tb03893.x

Bohnsack J.F., Brown E.J. 1986. The role of the spleen in resistance to infection. Annual Review of Medicine 37: 49-59. DOI: 10.1146/annurev.me.37.020186.000405

Bry C. 1982. Daily variations in plasma cortisol levels of individual female rainbow trout Salmo gairdneri: Evidence for a post-feeding peak in well-adapted fish. General and Comparative Endocrinology 48 (4): 462-468. DOI: 10.1016/0016-6480(82)90181-2

Capes S.E., Hunt D., Malmberg K., Pathak P., Gerstein H.C. 2001. Stress hyperglycemia and prognosis of stroke in nondiabetic and diabetic patients: A systematic overview. Stroke 32: 2426-2432. DOI: 10.1161/hs1001.096194 
Chaplin H.jr., Mollison P.L., Vetter H. 1953. The body/venous hematocrit ratio: its consistency over a wide hematocrit range. Journal of Clinical Investigation 32 (12): 1309-1316. DOI: 10.1172/JCI102859

Díaz M., Antonescu C.N., Capilla E., Klip A., Planas J.V. 2007. Fish glucose transporter (GLUT)-4 differs from rat GLUT4 in its Traffic characteristics but can translocate to the cell surface in response to insulin in skeletal muscle cells. Endocrinology 148 (11): 5248-5257. DOI: 10.1210/en.2007-0265

Gerwick L., Demers N.E., Bayne C.J. 1999. Modulation of stress hormones in rainbow trout by means of anesthesia, sensory deprivation and receptor blockade. Comparative Biochemistry and Physiology, Part A: Molecular and Integrative Physiology 124 (3): 329-334. DOI: 10.1016/S1095-6433(99)00126-9

Hadidi S., Glenney G.W., Welch T.J., Silverstein J.T., Wiens G.D. 2008. Spleen size predicts resistance of rainbow trout to Flavobacterium psychrophilum challenge. Journal of Immunology 180 (6): 4156-4165.

Holloway A.C., Keene J.L., Noakes D.G., Moccia R.D. 2004. Effects of clove oil and MS-222 on blood hormone profiles in rainbow trout Oncorhynchus mykiss, Walbaum. Aquaculture Research 35 (11): 1025-1030. DOI: 10.1111/j.1365-2109.2004.01108.x

Hsu F.-L., Liu I.-M., Kuo D.-H., Chen W.-C., Su H.-C., Cheng J.-T. 2003. Antihyperglycemic effect of puerarin in streptozotocin-induced diabetic rats. Journal of Natural Products 66 (6): 788-792. DOI: 10.1021/np0203887

Iwama G.K., Vijayan M.M., Forsyth R.B., Ackerman P.A. 1999. Heat shock proteins and physiological stress in fish. American Zoologist 39 (6): 901-909. DOI: 10.1093/icb/39.6.901

Laakso M., Edelman S.V., Brechtel G., Baron A.D. 1990. Decreased effect of insulin to stimulate skeletal muscle blood flow in obese man. A novel mechanism for insulin resistance. Journal of Clinical Investigation 85 (6): 1844-1852. DOI: 10.1172/JCI114644

Leonard J.B.K., McCormick S.D. 1999. Changes in haematology during upstream migration in American shad. Journal of Fish Biology 54 (6): 1218-1230. DOI: 10.1111/j.10958649.1999.tb02050.x

Meezan E., Meezan E.M., Jones K., Moore R., Barnes S., Prasain J. 2005. Contrasting effects of puerarin and daidzin on glucose homeostasis in mice. Journal of Agricultural and Food Chemistry 53 (22): 8760-8767. DOI: 10.1021/jf058105e

Moon T.W. 2001. Glucose intolerance in teleost fish: fact or fiction? Comparative Biochemistry and Physiology, Part B: Biochemistry and Molecular Biology 129 (2-3): 243-249. DOI: 10.1016/S1096-4959(01)00316-5

Mustafa A., MacKinnon B.M. 1999. Atlantic salmon, Salmo salar L., and Arctic char, Salvelinus alpinus (L.): comparative correlation between iodine-iodide supplementation, thyroid hormone levels, plasma cortisol levels, and infection intensity with the sea louse Caligus elongatus. Canadian Journal of Zoology 77 (7): 1092-1101. DOI: 10.1139/z99-060

Mustafa A., MacWilliams C., Fernandez N., Matchett K., Conboy G.A., Burka J.F. 2000. Effects of sea lice
(Lepeophtheirus salmonis Kröyer, 1837) infestation on macrophage functions in Atlantic salmon (Salmo salar L.). Fish and Shellfish Immunology 10 (1): 47- 59. DOI: 10.1006/fsim.1999.0229

Pearson M.P., Stevens E.D. 1991. Size and hematological impact of the splenic erythrocyte reservoir in rainbow trout, Oncorhynchus mykiss. Fish Physiology and Biochemistry 9 (1): 39-50. DOI: 10.1007/BF01987610

Peng N., Prasain J.K., Dai Y., Moore R., Arabshahi A., Barnes S., Carlson S., Wyss J.M. 2009. Chronic dietary kudzu isoflavones improve components of metabolic syndrome in stroke-prone spontaneously hypertensive rats. Journal of Agriculture and Food Chemistry 57 (16): 7268-7273. DOI: 10.1021/jf901169y

Poisot T., Šimková A., Hyršl P., Morand S. 2009. Interactions between immunocompetence, somatic condition and parasitism in the chub Leuciscus cephalus in early spring. Journal of Fish Biology 75 (7): 1667-1682. DOI: 10.1111/j.1095-8649.2009.02400.x

Prasain J.K., Kenneth J., Brissie N., Moore R., Wyss J.M., Barnes S. 2004. Identification of puerarin and its metabolites in rats by liquid chromatography-tandem mass spectrometry. Journal of Agricultural Food and Chemistry 52 (12): 3708-3712. DOI: 10.1021/jf040037t

Prasain J.K., Peng N., Rajbhandari R., Wyss J.M. 2012. The Chinese pueraria root extract (Pueraria lobata) ameliorates impaired glucose and lipid metabolism in obese mice. Phytomedicine 20 (1): 17-23. DOI: 10.1016/ j.phymed.2012.09.017

Ring C., Patterson S.M., Bacon S.L., Veldhuijzen van Zanten J.J.C.S., Willemsen G., Carroll D. 2008. Reliability of hematocrit during rest and stress in healthy adults. Biological Psychology 77 (1): 63-68. DOI: 10.1016/j.biopsycho.2007.09.005

Ruane N.M., Nolan D.T., Rotllant J., Costelloe J., Wendelaar Bonga S.E. 1999. Experimental exposure of rainbow trout Oncorhynchus mykiss (Walbaum) to the infective stages of the sea louse Lepeophtheirus salmonis (Krøyer) influences the physiological response to an acute stressor. Fish and Shellfish Immunology 10 (5): 451-463. DOI: $10.1006 /$ fsim.1999.0260

Tablin F., Chamberlain J.K., Weiss L. 2002. The microanatomy of the mammalian spleen. Pp. 11-21. In: Bowdler A.J. (ed.) The complete spleen: A handbook of structure, function, and clinical disorders. Humana Press, Totowa, NJ, USA.

Tanaka T., Tang H., Yu F., Michihara S., Uzawa Y., Zaima N., Moriyama T., Kawamura Y. 2011. Kudzu (Pueraria lobata) vine ethanol extracts improve ovariectomy-induced bone loss in female mice. Journal of Agricultural and Food Chemistry 59 (24): 13230-13237. DOI: 10.1021/jf2031617

Wang L.-H., Cheng Y.-Y. 2005. Solubility of puerarin in water, ethanol, and acetone from (288.2 to 328.2) K. Journal of Chemical Engineering Data 50 (4): 1375-1376. DOI: 10.1021/je050076g

Wedemeyer G.A., Barton B., McLeay D.J. 1990. Stress acclimation. Pp. 451-489. In: Schreck C.B., Moyle P.B. (eds.) Methods for fish biology. American Fisheries Society, Bethesda, MD, USA. 
Wendelaar Bonga S.E. 1997. The stress response in fish. Physiological Reviews 77 (3): 591-625.

Yan L.-P., Chan S.-W., Chan A.S.-C., Chen S.-L., Ma X.-J., Xu H.-X. 2006. Puerarin decreases serum total cholesterol and enhances thoracic aorta endothelial nitric oxide synthase expression in diet-induced hypercholesterolemic rats. Life Sciences 79 (4): 324-330. DOI: 10.1016/j.lfs.2006.01.016

Yeung D.K.Y., Leung S.W.S., Xu Y.C., Vanhoutte P.M., Man R.Y.K. 2006. Puerarin, an isoflavnoid derived from radix puerariae, potentiates endothelium-independent relaxation via the cyclic AMP pathway in porcine coronary artery. European Journal of Pharmacology 552 (1-3): 105-111. DOI: 10.1016/j.ejphar.2006.08.078

Yokoyama I., Ohtake T., Momomura S.-i., Yonekura K., Woo-Soo S., Nishikawa J., Sasaki J., Omata M. 1998. Hyperglycemia rather than insulin resistance is related to reduced coronary flow reserve in NIDDM. Diabetes 47: 119-124. DOI: 10.2337/diab.47.1.119

Received: 28 January 2013 Accepted: 1 April 2013 Published electronically: 30 June 2013 